\title{
Secular trends in serum lipid levels of a Middle Eastern adult population; 10 years follow up in Tehran lipid and glucose study
}

Masoumeh Kheirandish ${ }^{1}$, Samaneh Asgari ${ }^{1}$, Mojtaba Lotfaliany ${ }^{1}$, Mohammadreza Bozorgmanesh ${ }^{1}$, Navid Saadat ${ }^{1}$, Maryam Tohidi ${ }^{1}$, Fereidoun Azizi ${ }^{2}$ and Farzad Hadaegh ${ }^{1 *}$

\begin{abstract}
Background: To examine trends in the population levels of serum lipids among a Middle-Eastern adult population with high prevalence of dyslipidemia.

Methods: A population-based cohort of adult Iranian participants, aged $\geq 20$ years underwent four consecutive examinations between 1999-2001 and 2008-2011. Trends in age and multivariate-adjusted mean lipid levels were calculated using generalized estimating equations.

Results: At each of the 4 assessments, there were significant decreases in levels of total cholesterol (TC) (multivariate-adjusted means, 5.21 vs. $4.88 \mathrm{mmol} / \mathrm{L}$ in men; $5.42 \mathrm{vs.} 5.07 \mathrm{mmol} / \mathrm{L}$ in women), triglycerides (TGs) (2.11 vs. $1.94 \mathrm{mmol} / \mathrm{L}$ in men; 1.88 vs. $1.74 \mathrm{mmol} / \mathrm{L}$ in women), and an increase in HDL-C level in both genders (0.95 vs. $1.058 \mathrm{mmol} / \mathrm{L}$ in men; $1.103 \mathrm{vs} .1 .246 \mathrm{mmol} / \mathrm{L}$ in women) in multivariate analyses (all Ps <0.001); however, body mass index (BMI) significantly increased simultaneously (25.92 vs. $27.45 \mathrm{~kg} / \mathrm{m} 2$ in men; $27.76 \mathrm{vs.} 30.02 \mathrm{~kg} / \mathrm{m} 2 \mathrm{in}$ women) $(P<0.001)$. There were significant $(P<0.001)$ increases in fasting plasma glucose $(F P G)$ levels only among men (5.35 vs. $5.73 \mathrm{mmol} / \mathrm{L}$ ). Results did not change after excluding participants that had cardiovascular disease or used lipid lowering drugs during follow-up. There were significant decreases in the prevalence of hypercholesterolemia, low HDL-C, hypertriglyceridemia (all Ps <0.001) during follow-up. Furthermore, the consumption of lipid lowering drugs significantly increased $(P<0.001)$.

Conclusion: During a 10 years follow-up, favorable trends were observed in the population levels of TC, triglycerides, HDL-C, which could not be fully accounted for by the increase observed in the consumption of lipid lowering drugs. These favorable trends were counterbalanced by the progressive increase in general obesity and FPG level.
\end{abstract}

Keywords: Secular trends, Serum lipids measures, Middle Eastern adult population, Cohort, Dislipidemia

\section{Background}

Coronary heart disease (CHD) is one of the main causes of mortality and morbidity worldwide, leading severe concern that CHD will become pandemic problem [1]. Cardiovascular disease (CVD) account for $38-50 \%$ of deaths in Iran [2]. Numerous studies have reported that high serum cholesterol and low high density lipoprotein

\footnotetext{
* Correspondence: fzhadaegh@endocrine.ac.ir

'Prevention of Metabolic Disorders Research Center, Research Institute for Endocrine Science, Shahid Beheshti University of Medical Sciences, \#24, Parvaneh st, Yemen st, Chamran Exp, P.O. Box: 19395-4763, Tehran, Islamic Republic of Iran

Full list of author information is available at the end of the article
}

cholesterol (HDL-C) are major risk factors for coronary heart disease $[3,4]$. There are also evidences to show that high triglycerides and CHD have an association $[5,6]$. The prevalence of dyslipidemia between Tehranian adult population has been reported to be high [7]. We have recently observed that all lipid measures were significant predictors of incident CHD among an Iranian population aged $\geq 50$ years in sex and multivariate-adjusted regression models [8].

Trends in a lipid measure vary across different countries; even in the same country different lipid measures might have different trends in both 
directions and magnitudes of changes. A number of studies have examined the trends in serum lipid levels either in repeated cross-sectional time-series [9-15] or in longitudinal cohort studies [16-18]. It seems that the trends in the levels of total and low density lipoprotein cholesterol (LDL-C) are favorable in most of countries, except India, China and Japan $[9,10,12]$. In a global study of trends in serum total cholesterol in 199 countries, the mean level of total cholesterol changed little between 1980 and 2008, declining by less than $0.1 \mathrm{mmol} / \mathrm{L}$ per decade in men and women. Total cholesterol decreased in the high-income region consisting of Australasia, North America, and western Europe, and in central and eastern Europe while it increased in east and southeast Asia and Pacific [19]. In a previous report on time-trends in lipid measures among an Iranian adult population, we observed short-term favorable trends paralleling the increasing trend in obesity measures, whether such favorable trends have extended to a longer time-frame in the light of increases observed in the diabetes and obesity remains to be elucidated $[15,20]$.

Recently the fourth follow-up assessment of a large community-based longitudinal study of a Middle East population, the Tehran Lipid and Glucose Study (TLGS) has been completed, herein, the trends in the population levels of serum lipids and indices as well as trends in prevalence of dyslipidemia among TLGS' participants followed for more than a decade, enabling us to investigate the trends in lipid measures during the last decade.

\section{Methods}

\section{Study design and sample}

Detailed descriptions of TLGS have been reported elsewhere [21]. In brief, the TLGS is a large scale, long term, community-based prospective study performed on a representative sample of residents of district 13 of Tehran, the capital of Iran. The TLGS has two major components: a cross-sectional prevalence study of noncommunicable disease and associated risk factors, implemented between March 1999 and December 2001, and a

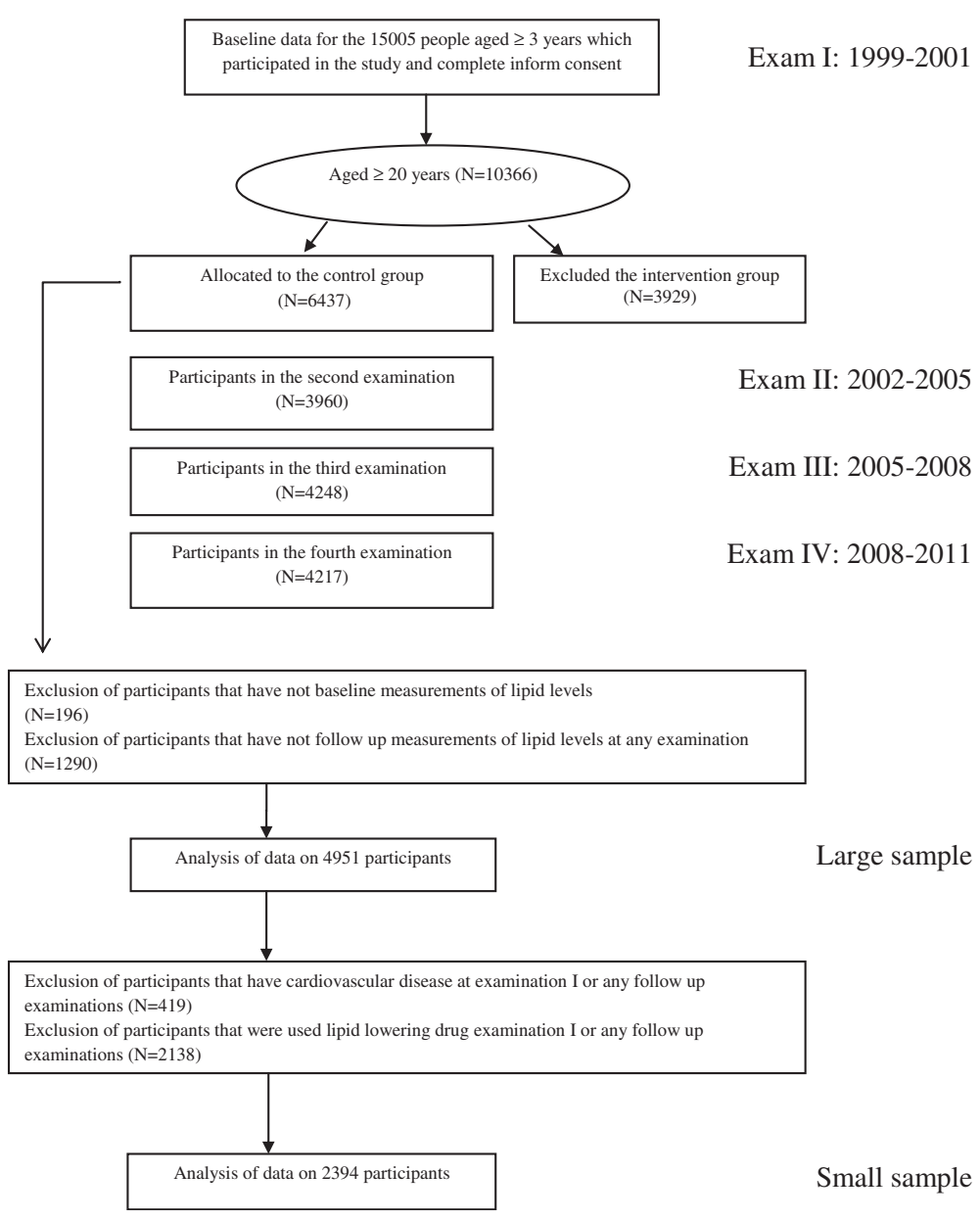

Figure 1 Outline of selection design of study participants. 
prospective follow-up study. Data collection is ongoing, designed to continue for at least 20 years, on triennial basis.

\section{Study population}

Of total of 27340 residents aged $\geq 3$ years invited by telephone call, 15005 residents participated in first examination. Of this population those aged $\geq 20$ years $(n=10366)$, were categorized into the cohort $(n=6437)$ and intervention group $(n=3929)$, the latter to be educated for implementation of life style modifications. After excluding intervention group, participants without any lipid levels record in baseline $(n=196)$, and participants without follow-up record in any examination $(n=1290)$, the final sample consisted of a total of 4951 individuals (2866 women) with at least 1 follow-up. In the secondary analyses, we analyzed trends of lipid levels in a smaller sample (2394) obtained by excluding individuals with prevalent or incident cardiovascular disease (CVD) and current use of lipid lowering drugs (Figure 1).

The design of the study was approved by the Ethics Committee of the Research Institute for Endocrine Sciences, Shahid Beheshti University of Medical Sciences, and all participants provided written informed consent.

\section{Clinical and anthropometric measurements}

Subjects were interviewed by trained interviewers using pretested questionnaires. Information on age, sex, past medical history of CVD, medication use, smoking habits and family history of premature CVD was collected. Anthropometric measured including weight and waist circumference (WC). Using standard protocols, WC was measured by a trained individual at the level of the umbilicus. Body mass index (BMI) was calculated as weight in kilograms divided by height in squared meters. Blood pressure (BP) was measured twice in a seated position after $15 \mathrm{~min}$ resting using a standard mercury sphygmomanometer. Education status was categorized into 3 groups: 1. Illiterate/primary school (less than 6 years); 2. Below diploma /diploma (6-12 years) and 3 . Higher than diploma (more than 12 years). Marital status was categorized as single, married and widowed/divorced.

\section{Laboratory measurements}

After 12-14 h overnight fasting, blood samples were drawn from veins of the participitants into Vacutainer ${ }^{\circledR}$ tubes between 7.00 and 9.00 A.M. and centrifuged within 30-45 min of collection. Fasting plasma glucose (FPG) was measured by the enzymatic colorimetric glucose oxidase method; inter-and intra-assay coefficients of variation $(\mathrm{CV})$

Table 1 Baseline characteristics comparison of participants with and without follow-up with respect to different genders*

\begin{tabular}{|c|c|c|c|c|c|c|}
\hline \multirow{2}{*}{ 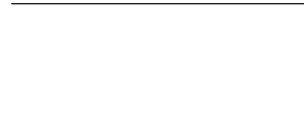 } & \multicolumn{2}{|l|}{ Male } & \multirow[b]{2}{*}{$\mathrm{p}$-value } & \multicolumn{2}{|l|}{ Female } & \multirow[b]{2}{*}{ p-value } \\
\hline & $\begin{array}{l}\text { Follow up } \\
(\mathrm{N}=3427)\end{array}$ & $\begin{array}{l}\text { No follow up } \\
(\mathrm{N}=968)\end{array}$ & & $\begin{array}{l}\text { Follow up } \\
(\mathrm{N}=4687)\end{array}$ & $\begin{array}{l}\text { No follow up } \\
(\mathrm{N}=1284)\end{array}$ & \\
\hline Age (years) & $44.03(15.4)$ & $44.46(17.37)$ & 0.5 & $41.61(13.85)$ & $42.23(16.4)$ & 0.22 \\
\hline Education $^{\ddagger}$ & & & 0.6 & & & 0.43 \\
\hline$<6$ yrs & $971(28.4)$ & $290(30)$ & & $1909(40.8)$ & $540(42.1)$ & \\
\hline 6-12 yrs & $1870(54.6)$ & $513(53.1)$ & & $2373(50.7)$ & $625(48.8)$ & \\
\hline$>12$ yrs & $582(17)$ & $164(17)$ & & $397(8.5)$ & $117(9.1)$ & \\
\hline Marital status ${ }^{\ddagger}$ & & & 0.01 & & & $<0.001$ \\
\hline Single & $570(16.6)$ & $202(20.9)$ & & $496(10.6)$ & $197(15.3)$ & \\
\hline Married & $2829(82.6)$ & $758(78.3)$ & & $3755(80.1)$ & $928(72.3)$ & \\
\hline Divorce or widow & $28(0.8)$ & $8(0.8)$ & & $436(9.3)$ & $159(12.4)$ & \\
\hline Current smoking $^{\ddagger}$ & $923(27.5)$ & $319(33.8)$ & $<0.001$ & $162(3.5)$ & $72(5.7)$ & $<0.001$ \\
\hline BMI $\left(\mathrm{kg} / \mathrm{m}^{2}\right)$ & $25.88(4.07)$ & $25.46(4.25)$ & 0.006 & $27.40(5.38)$ & $27.16(5.83)$ & 0.22 \\
\hline Triglycerides $(\mathrm{mmol} / \mathrm{L})^{+}$ & $156(119)$ & $139(109)$ & $<0.001$ & $137(112)$ & $136(110)$ & 0.5 \\
\hline HDL-C (mmol/L) & $38.42(9.36)$ & $38.36(9.80)$ & 0.87 & $45.06(11.14)$ & $45.02(11.42)$ & 0.92 \\
\hline Cholesterol (mmol/L) & $205.45(43.34)$ & $200.73(42.68)$ & 0.004 & 212.95 (48.9) & $212.52(50.84)$ & 0.78 \\
\hline
\end{tabular}

Abbreviations: BMI body mass, HDL-C high-density lipoprotein cholesterol, TG triglycerides.

*Values are presented as mean (SD) for continuous parameters.

${ }^{\dagger}$ Median (IQ).

${ }^{\ddagger} \mathrm{n}(\%)$. 
at baseline and follow-up phases were both less than $2.3 \%$. A Selectra 2 autoanalyzer (Vital Scientific, Spankeren, Netherlands) was used in the TLGS research laboratory, on the day of blood collection, to analyze samples for serum total cholesterol (TC) and TGs. Enzymatic colorimetric tests were used to assay TC with cholesterol esterase and cholesterol oxidase; for TGs, glycerol phosphate oxidase was used. HDL-C was measured after precipitation of the lipoprotein-B-containing lipoproteins with phosphotungstic acid. LDL-C was calculated according to the Friedewald formula if TGs were $<4.5 \mathrm{mmol} / \mathrm{l}$ [22]. Non-HDL-C was calculated by subtracting HDL-C from TC; TC/HDL-C and TG/HDL-C were calculated by dividing TC and TG by HDL-C, respectively. Both inter and intra-assay coefficients of variation were less than 1.9, 2.1 and 3\% for TC, TGs and HDL-C, respectively in all baseline and follow-up assays of lipid profile.

\section{Cardiovascular disease outcome}

Details of the collection of cardiovascular outcome data have been published elsewhere [21]. In the current study, the events targeted were the first CVD events, including definite myocardial infarction (MI), probable MI, unstable angina, angiographic-proven coronary heart disease (CHD), and stroke (as defined by a new neurological deficit that lasted more than $24 \mathrm{~h}$ ).

\section{Definition of terms}

Dyslipidemia was defined as follows: Hypercholesterolemia: serum $\mathrm{TC} \geq 6.19 \mathrm{mmol} / \mathrm{L}$; Hypertriglyceridemia: serum TGs $\geq 2.26 \mathrm{mmol} / \mathrm{L}$; Low HDL-C: serum HDL < $1.036 \mathrm{mmol} / \mathrm{L}$; High non-HDL-C: serum non-HDL-C $\geq$ $5.15 \mathrm{mmol} / \mathrm{L}$; High TC/HDL-C $\geq 5.97$; High TG /HDL$\mathrm{C} \geq 2.18$ [23].

Participants who had systolic blood pressure $\geq 140$ $\mathrm{mmHg}$ or diastolic blood pressure $\geq 90 \mathrm{mmHg}$ or were on antihypertensive drugs were referred to as hypertensive. Diabetes mellitus was ascertained among participants who had FPG $\geq 7 \mathrm{mmol} / \mathrm{L}$ or were on glucose lowering medication. Participants who were smoking at the time of examination were considered as current smokers.

\section{Statistical analysis}

Continuously-distributed variables were described by reporting their mean (SD). Median and inter-quartile

Table 2 Baseline characteristics participants by gender

\begin{tabular}{|c|c|c|c|}
\hline & Male $(\mathrm{N}=2085)$ & Female $(\mathrm{N}=2866)$ & $\overline{p \text {-value }}$ \\
\hline Age (years) & $43.8(15.011)$ & $41.61(13.77)$ & $<0.001$ \\
\hline Education $^{\neq}$ & & & $<0.001$ \\
\hline$<6 \mathrm{yrs}$ & $566(27.2)$ & $1130(39.5)$ & \\
\hline $6-12$ yrs & $1148(55.1)$ & $1479(51.7)$ & \\
\hline$>12$ yrs & $368(17.7)$ & $251(8.8)$ & \\
\hline Marital status $^{\ddagger}$ & & & $<0.001$ \\
\hline Single & $362(17.4)$ & $283(9.9)$ & \\
\hline Married & 1709 (82.0) & $2330(81.3)$ & \\
\hline Divorce or widow & $14(0.7)$ & $253(8.8)$ & \\
\hline Current smoking $^{\ddagger}$ & $568(27.9)$ & $118(4.2)$ & $<0.001$ \\
\hline BMI $\left(\mathrm{kg} / \mathrm{m}^{2}\right)$ & $25.73(4.046)$ & $27.51(5.2)$ & $<0.001$ \\
\hline Waist circumference (cm) & $88.53(11.33)$ & $87.62(12.8)$ & 0.011 \\
\hline Systolic BP (mm Hg) & $120.71(18.51)$ & $118.45(18.95)$ & $<0.001$ \\
\hline Diastolic BP (mm Hg) & $78(11.02)$ & $77.82(10.43)$ & 0.6 \\
\hline $\mathrm{TC}(\mathrm{mmol} / \mathrm{L})$ & $5.3(1.124)$ & $5.51(1.28)$ & $<0.001$ \\
\hline $\mathrm{HDL}-\mathrm{C}(\mathrm{mmol} / \mathrm{L})$ & $1.0(0.24)$ & $1.166(0.30)$ & $<0.001$ \\
\hline $\mathrm{TG}(\mathrm{mmol} / \mathrm{L})^{\dagger}$ & $1.75(1.31)$ & $1.54(1.24)$ & $<0.001$ \\
\hline Non-HDL-C (mmol/L) & $4.3(1.13)$ & $4.34(1.28)$ & 0.252 \\
\hline $\mathrm{TC} / \mathrm{HDI}-\mathrm{C}$ & $5.6(1.75)$ & $5.0(1.53)$ & $<0.001$ \\
\hline $\mathrm{TG} / \mathrm{HDL}-\mathrm{C}^{\dagger}$ & $1.82(1.06)$ & $1.35(1.34)$ & $<0.001$ \\
\hline FPG (mmol/L) & $5.38(1.5)$ & $5.45(1.97)$ & 0.13 \\
\hline
\end{tabular}

Abbreviations: BMI body mass, BP blood pressure, FPG Fasting plasma glucose, HDL-C high-density lipoprotein cholesterol, non HDL-C non high-density lipoprotein cholesterol, $T C$ total cholesterol, TG triglycerides.

*Values are presented as mean (SD) for continuous parameters.

${ }^{\dagger}$ Median (IQ)

${ }^{\ddagger} \mathrm{n}(\%)$. 
ranges were reported for TGs levels because the distribution was highly positively skewed. All analyses were performed separately for males and females. The statistical significance of the differences in mean levels of normally distributed variables was examined using the t-test. The Kruskal-Wallis test was used for TGs. Chisquare test was performed to test univariate statistical association between categorical variables at baseline.

All baseline characteristics including age, educational level, marital status, smoking, systolic blood pressure, WC, diabetes, and history of CVD, were included in the logistic model with participation as the outcome. The probability of participation was estimated using logistic model and used as a propensity score. We added this propensity score to the longitudinal models as a covariate and examined if the probability of participation was associated with trend in lipid levels [24]. Furthermore we adjusted propensity score for all baseline characteristics in all four examination cycles and the parameter estimates remained essentially unchanged. Therefore, the selection bias is unlikely to have affected our estimations.

Trends of TC, LDL-C, HDL-C, and TGs levels were primary lipid measures of interest for the current

Table 3 Characteristics of participants by gender at examination cycle (baseline +3 follow ups)

\begin{tabular}{|c|c|c|c|c|}
\hline & Exam 1 (1999-2001) & Exam 2 (2002-2005) & Exam 3 (2005-2008) & Exam 4 (2008-2011) \\
\hline \multicolumn{5}{|l|}{ Male } \\
\hline Age (years) & $43.8(15.011)$ & $47.50(14.15)$ & $50.57(14.14)$ & $54.00(14.16)$ \\
\hline BMI $\left(\mathrm{kg} / \mathrm{m}^{2}\right)$ & $25.73(4.046)$ & $26.57(5.22)$ & $27(5.25)$ & $27.23(6.4)$ \\
\hline WC (cm) & $88.53(11.33)$ & $94.60(10.65)$ & $96.18(10.23)$ & $97.52(10.76)$ \\
\hline Systolic BP (mm Hg) & $120.71(18.51)$ & $119.2(17.55)$ & $120.93(18.91)$ & $122.73(18.26)$ \\
\hline Diastolic BP $(\mathrm{mm} \mathrm{Hg})$ & $78(11.02)$ & 75.58 (10.91) & $76.57(9.97)$ & $79.91(10.92)$ \\
\hline $\mathrm{TC}(\mathrm{mmol} / \mathrm{L})$ & $5.3(1.124)$ & $5.02(0.99)$ & $4.97(0.96)$ & $4.96(1.05)$ \\
\hline HDL-C (mmol/L) & $1.0(0.24)$ & $0.9(0.21)$ & $0.96(0.21)$ & $1.11(0.24)$ \\
\hline $\mathrm{TG}(\mathrm{mmol} / \mathrm{L})^{\dagger}$ & $1.75(1.31)$ & $2.05(1.38)$ & $2.01(1.23)$ & $1.9(1.37)$ \\
\hline Non-HDL-C (mmol/L) & $4.3(1.13)$ & $4.11(0.96)$ & $4.00(0.95)$ & $3.83(0.96)$ \\
\hline TC/HDL-C & $5.6(1.75)$ & $5.83(1.63)$ & $5.40(1.5)$ & $4.63(1.35)$ \\
\hline $\mathrm{TG} / \mathrm{HDL}-\mathrm{C}^{\dagger}$ & $1.82(1.06)$ & $2.50(1.86)$ & $2.29(1.7)$ & $1.86(1.46)$ \\
\hline FPG (mmol/L) & $5.38(1.5)$ & $5.56(1.58)$ & $5.51(1.64)$ & $5.86(1.8)$ \\
\hline Current smoking $^{\ddagger}$ & $568(27.9)$ & $489(23.5)$ & $553(26.5)$ & $555(26.6)$ \\
\hline Lipid drug $^{\ddagger}$ & $34(1.66)$ & $49(3.2)$ & $62(3.77)$ & $164(9.63)$ \\
\hline \multicolumn{5}{|l|}{ Female } \\
\hline Age (year) & $41.61(13.77)$ & $45.5(13.21)$ & $48.46(13.13)$ & $51.95(13.15)$ \\
\hline BMI $\left(\mathrm{kg} / \mathrm{m}^{2}\right)$ & $27.51(5.2)$ & $29(4.8)$ & $29.2(4.8)$ & $30.23(15)$ \\
\hline$W C(\mathrm{~cm})$ & $87.62(12.8)$ & $92.17(12.28)$ & $91.71(12.36)$ & $96.5(12)$ \\
\hline Systolic BP (mm Hg) & 118.45 (18.95) & $116.9(19.42)$ & 115.18 (19.92) & $118.87(21.08)$ \\
\hline Diastolic BP $(\mathrm{mm} \mathrm{Hg})$ & $77.82(10.43)$ & $75.51(10.18)$ & 73.05 (10.34) & $76.68(11.14)$ \\
\hline $\mathrm{TC}(\mathrm{mmol} / \mathrm{L})$ & $5.51(1.28)$ & $5.23(1.14)$ & $5.17(1.07)$ & $5.2(1.08)$ \\
\hline $\mathrm{HDL}-\mathrm{C}(\mathrm{mmol} / \mathrm{L})$ & $1.166(0.30)$ & $1.05(0.26)$ & $1.13(0.27)$ & $1.31(0.28)$ \\
\hline TG $(\mathrm{mmol} / \mathrm{L})^{\dagger}$ & $1.54(1.24)$ & $1.85(1.16)$ & $1.81(1.06)$ & $1.73(0.96)$ \\
\hline Non-HDL-C (mmol/L) & $4.34(1.28)$ & $4.17(1.13)$ & $4.04(1.46)$ & $3.88(1.08)$ \\
\hline TC/HDL-C & $5.0(1.53)$ & $5.24(1.7)$ & $4.8(1.46)$ & $4.11(1.19)$ \\
\hline TG/HDL-C ${ }^{+}$ & $1.35(1.34)$ & $1.97(1.63)$ & $1.8(1.38)$ & $1.45(1.07)$ \\
\hline FPG (mmol/L) & $5.45(1.97)$ & $5.57(1.86)$ & $5.55(1.96)$ & $5.9(2.08)$ \\
\hline Current smoking $^{\ddagger}$ & $118(4.2)$ & $159(5.5)$ & $141(4.9)$ & $160(5.6)$ \\
\hline Lipid drug ${ }^{\ddagger}$ & $131(4.64)$ & 109 (4.83) & $152(6.5)$ & $296(12.38)$ \\
\hline
\end{tabular}

Abbreviations: $B M I$ body mass index, BP blood pressure, Exam examination cycle, FPG Fasting plasma glucose, $T C$ total cholesterol, $H D L-C$ high-density lipoprotein cholesterol, non HDL-C non-high-density lipoprotein cholesterol, TG triglycerides, WC waist circumference.

*Values are presented as mean (SD) unless otherwise indicated.

tMedian (IQ).

${ }^{\ddagger} \mathrm{n}(\%)$. 
analyses. We further explored the trend in the population levels of non-HDL-C, TC/HDL-C and TG/HDL-C ratio as well. Time trends in the population levels of the lipid measures, were examined using generalized estimating equations (GEE). The GEE method facilitates analysis of longitudinal data or repeated measures on dependent variables of many different distributions, mainly binary data [25]. GEE is a statistical technique enabling researchers to restrict modeling to the first moment which require only the correct working correlation matrix which specified the univariate marginal distributions [26]. Analysis with an exchangeable correlation structure with only random intercept was designed for the current data. Such an approach enabled us to account for the correlation among observations following assessment of each examination cycle. Models were developed at two hierarchial levels; age-adjusted (adjusting for age, propensity score and examination cycle) and multivariate-adjusted (adjusting for age, examination cycle, propensity score, BMI, current smoking, diabetes, hypertension and total cholesterol level [in analysis of HDL-C and TG]). Trends in BMI and FPG were also examined in both age and multivariate-adjusted models.

We also compared the trends in prevalence of high cholesterol, high TGs, low HDL-C, high non-HDL-C, high TC/HDL-C and high TG/HDL-C. The significance of trends in the proportions was examined by pooled logistic regression. All the analyses were repeated in the smaller sample (i.e. excluding individuals with prevalent or incident CVD and current use of lipid lowering drugs).

P-values $<0.05$ were considered significant. All analyses were performed using SAS statistical software (ver. 9.2, SAS Institute, Car, NC, USA).

\section{Results}

The comparison between baseline characteristics of participants with and without follow-up disclosed that the followed men had higher mean levels of TC, TGs and BMI, although they had lower rate of smoking. There was no difference in the lipid status between the followed versus non followed women; however, the followed participants had lower rate of smoking (Table 1).

In the baseline examination, male participants were older, more likely to be single, being smokers, and had higher educational levels, as well as higher TGs, TC/ HDL-C and TG/HDL-C levels compared to female participants; however mean BMI, total and HDL-C levels were higher in female participants in comparison with male ones (Table 2).

Table 4 Age-adjusted mean levels* of fasting lipids, FPG and BMI by examination cycle in large sample

\begin{tabular}{|c|c|c|c|c|c|}
\hline & Exam 1 (1999-2001) & Exam 2 (2002-2005) & Exam 3 (2005-2008) & Exam 4 (2008-2011) & $p$-value for trend \\
\hline \multicolumn{6}{|l|}{ Male } \\
\hline $\mathrm{TC}^{+}$ & $5.22(5.21-5.23)$ & $5.12(5.10-5.13)$ & $4.99(4.95-5.00)$ & $4.88(4.87-4.90)$ & $<0.001$ \\
\hline HDL-C ${ }^{\dagger}$ & $0.943(0.942-0.944)$ & $0.982(0.981-0.983)$ & $1.016(1.015-1.017)$ & $1.052(1.051-1.053)$ & $<0.001$ \\
\hline $\mathrm{TG}^{\dagger}$ & $2.08(2.07-2.09)$ & $2.01(2.00-2.02)$ & $1.96(1.95-1.97)$ & $1.90(1.89-1.91)$ & $<0.001$ \\
\hline Non-HDL-C ${ }^{\dagger}$ & $4.28(4.27-4.29)$ & $4.13(4.12-4.14)$ & 3.97 (3.96-3.98) & $3.82(3.81-3.83)$ & $<0.001$ \\
\hline TG/HDL-C & $2.45(2.44-2.47)$ & $2.28(2.27-2.30)$ & $2.15(2.14-2.16)$ & $2.00(1.99-2.01)$ & $<0.001$ \\
\hline TC /HDL-C & $5.81(5.80-5.81)$ & $5.48(5.47-5.50)$ & $5.17(5.16-5.18)$ & $4.85(4.84-4.86)$ & $<0.001$ \\
\hline $\mathrm{FPG}^{\dagger}$ & $5.35(5.32-5.37)$ & $5.54(5.51-5.57)$ & $5.62(5.59-5.64)$ & $5.73(5.71-5.75)$ & $<0.001$ \\
\hline BMI $\left(k g / m^{2}\right)$ & $25.88(25.85-25.92)$ & $26.36(26.32-26.4)$ & $26.87(26.83-26.9)$ & $27.37(27.33-27.40)$ & $<0.001$ \\
\hline \multicolumn{6}{|l|}{ Female } \\
\hline $\mathrm{TC}^{\dagger}$ & $5.42(5.40-5.44)$ & $5.35(5.33-5.37)$ & $5.19(5.17-5.21)$ & $5.07(5.05-5.09)$ & $<0.001$ \\
\hline HDL-C ${ }^{\dagger}$ & 1.1004 (1.1002-1.1007) & $1.1486(1.1484-1.1487)$ & $1.1960(1.195-1.197)$ & $1.2450(1.244-1.246)$ & $<0.001$ \\
\hline $\mathrm{TG}^{\dagger}$ & $1.86(1.84-1.87)$ & $1.84(1.83-1.86)$ & $1.76(1.75-1.77)$ & $1.71(1.69-1.72)$ & $<0.001$ \\
\hline Non-HDL-C ${ }^{\dagger}$ & $4.32(4.30-4.34)$ & $4.2(4.18-4.22)$ & $4(3.98-4.02)$ & $3.83(3.81-3.85)$ & $<0.001$ \\
\hline TG/HDL-C & $1.9(1.88-1.91)$ & $1.81(1.80-1.82)$ & $1.66(1.64-1.70)$ & $1.53(1.52-1.54)$ & $<0.001$ \\
\hline $\mathrm{TC} / \mathrm{HDL}-\mathrm{C}$ & $5.19(5.17-5.21)$ & $4.93(4.91-4.95)$ & $4.6(4.57-4.61)$ & $4.28(4.26-4.30)$ & $<0.001$ \\
\hline $\mathrm{FPG}^{\dagger}$ & $5.47(5.44-5.49)$ & $5.63(5.60-5.66))$ & $5.63(5.61-5.66)$ & $5.78(5.75-5.81)$ & 0.37 \\
\hline BMI $\left(k g / m^{2}\right)$ & $27.73(27.63-27.78)$ & $26.64(28.57-28.7)$ & 29.24 (29.18-29.3) & 29.94 (29.88-30.0) & $<0.001$ \\
\hline
\end{tabular}

Abbreviations: BMI body mass index, Exam examination cycle, FPG fasting plasma glucose, $H D L-C$ high-density lipoprotein cholesterol, non $H D L-C$ non-high-density lipoprotein cholesterol, TC total cholesterol, TG triglycerides.

*Values are age adjusted means (95\% confidence intervals) from generalized estimating equations to account for correlated observations. Adjusted for age, propensity score and examination cycle.

${ }^{\dagger} \mathrm{mmol} / \mathrm{L}$. 
The baseline as well as follow-up characteristics of three assessments are shown in Table 3 in each gender. At baseline examination the mean age of participants were 43.8 (15.011) and 41.61 (13.77) years for men and women, respectively. Mean BMI was 25.73 (4.046) and $27.51(5.2) \mathrm{kg} / \mathrm{m}^{2}$ in men and women, respectively.

As presented in the Tables 4 and 5, we observed a statistically significant decrease in the age and multivariate-adjusted levels of total and non-HDL cholesterol, TC/HDL-C and TG/HDL-C, as well as TGs for both genders ( $p$-value for TGs trend was marginally significant for men). In contrast there was a significant increase in HDL-C levels across followup examinations in both men and women (all Pvalues $<0.001)$. Meanwhile, the results demonstrated a significant increase in the population levels of BMI (in both genders) and FPG (only among men) in the same time frame in both genders.

Table 6 show the time trends in the age and multivariate-adjusted prevalence rate of different aspects of dyslipidemia in the large sample. During the study, in both gender, there were significant decreases in the prevalence of hypercholesterolemia, hypertriglyceridemia, low HDL-C, high non-HDL-C, high TC/HDL-C and high TG/HDL-C. Furthermore consumption of lipid lowering drugs significantly $(\mathrm{p}<0.001)$ increased in both genders.

As ancillary analyses we repeated all analyses among individuals without CVD who were not on lipid lowering drugs and observed that the results remained essentially unchanged in both age and multivariate-adjusted models (Tables 7, 8 and 9).

\section{Discussion}

Using a large cohort of a Middle Eastern adults population, we demonstrated favorable time trends in the population levels of total cholesterol, HDL-C, non-HDL-C, TG, TG/HDL-C and TC/HDL-C among both genders during 10 years follow up. Such favorable trends in lipids levels and ratios could not be fully accounted for by the significant increase in consumption of lipid lowering drugs. In contrast, however, population levels of general adiposity and FPG have increased in both genders potentially leading to long term risk of cardiovascular disease.

Table 5 Multivariate-adjusted mean levels* of fasting lipids, FPG and BMI by examination cycle in large sample

\begin{tabular}{|c|c|c|c|c|c|}
\hline & Exam 1 (1999-2001) & Exam 2 (2002-2005) & Exam 3 (2005-2008) & Exam 4 (2008-2011) & $p$-value for trend \\
\hline \multicolumn{6}{|l|}{ Male } \\
\hline $\mathrm{TC}^{+}$ & $5.21(5.20-5.22)$ & $5.13(5.12-5.15)$ & $5.00(4.99-5.01)$ & $4.88(4.87-5.00)$ & $<0.001$ \\
\hline LDL-C $^{+}$ & $3.82(3.81-3.83)$ & $3.73(3.72-3.74)$ & $3.58(3.57-3.59)$ & $3.43(3.42-3.45)$ & $<0.001$ \\
\hline HDL-C ${ }^{\dagger}$ & $0.95(0.94-0.95)$ & $0.97(0.96-0.98)$ & $1.013(1.010-1.017)$ & $1.058(1.055-1.062)$ & $<0.001$ \\
\hline $\mathrm{TG}^{+}$ & $2.11(2.08-2.14)$ & $1.96(1.92-1.99)$ & $1.93(1.90-1.96)$ & $1.94(1.91-1.98)$ & 0.057 \\
\hline Non-HDL-C $C^{\dagger}$ & $4.26(4.25-4.28)$ & $4.15(4.14-4.17)$ & $3.98(3.97-4.0)$ & $3.82(3.80-3.83)$ & $<0.001$ \\
\hline TG/HDL & $2.43(2.41-2.46)$ & $2.31(2.3-2.34)$ & $2.15(2.12-2.17)$ & $2.00(1.97-2.03)$ & $<0.001$ \\
\hline $\mathrm{TC} / \mathrm{HDL}$ & $5.77(5.75-5.8)$ & $5.52(5.5-5.54)$ & $5.18(5.16-5.2)$ & $4.84(4.81-4.86)$ & $<0.001$ \\
\hline $\mathrm{FPG}^{+}$ & $5.35(5.32-5.37)$ & $5.54(5.51-5.57))$ & $5.62(5.59-5.64)$ & $5.73(5.71-5.75)$ & $<0.001$ \\
\hline BMI $\left(\mathrm{kg} / \mathrm{m}^{2}\right)$ & $25.92(25.87-25.97)$ & $26.3(26.23-26.35)$ & $26.78(26.72-26.84)$ & $27.45(27.4-27.51)$ & $<0.001$ \\
\hline \multicolumn{6}{|l|}{ Female } \\
\hline $\mathrm{TC}^{+}$ & $5.42(5.39-5.43)$ & $5.37(5.34-5.4)$ & $5.18(5.16-5.20)$ & $5.07(5.04-5.06)$ & $<0.001$ \\
\hline LDL-C ${ }^{+}$ & $3.90(3.88-3.92)$ & $3.81(3.79-3.83)$ & $3.61(3.59-3.63)$ & $3.46(3.44-3.48)$ & $<0.001$ \\
\hline HDL-C ${ }^{\dagger}$ & $1.103(1.101-1.104)$ & $1.140(1.130-1.142)$ & $1.039(1.037-1.194)$ & $1.246(1.241-1.251)$ & $<0.001$ \\
\hline $\mathrm{TG}^{+}$ & $1.88(1.86-1.91)$ & $1.80(1.77-1.83)$ & $1.71(1.68-1.73)$ & $1.74(1.71-1.77)$ & $<0.001$ \\
\hline Non-HDL-C ${ }^{\dagger}$ & $4.31(4.29-4.33)$ & $4.22(4.20-4.24)$ & $3.98(3.96-4.00)$ & $3.82(3.80-3.85)$ & $<0.001$ \\
\hline TG/HDL-C & 1.88 (1.87-1.90) & $1.82(1.80-1.84)$ & $1.63(1.61-1.65)$ & $1.54(1.51-1.57)$ & $<0.001$ \\
\hline TC/HDL-C & $5.20(5.17-5.21)$ & $4.95(4.93-4.98)$ & $4.57(4.56-4.60)$ & $4.28(4.25-4.32)$ & $<0.001$ \\
\hline $\mathrm{FPG}^{+}$ & $5.47(5.44-5.49)$ & $5.63(5.60-5.66)$ & $5.63(5.61-5.66)$ & $5.78(5.75-5.81)$ & 0.37 \\
\hline BMI $\left(\mathrm{kg} / \mathrm{m}^{2}\right)$ & $27.76(27.7-27.82)$ & $28.64(28.56-28.71)$ & $29.11(29.05-29.18)$ & $30.02(29.95-30.10)$ & $<0.001$ \\
\hline
\end{tabular}

Abbreviations: BMI body mass index, Exam examination cycle, FPG fasting plasma glucose, $H D L-C$ high-density lipoprotein cholesterol, $L D L-C$ low-density lipoprotein cholesterol, non HDL-C non-high-density lipoprotein cholesterol, TC total cholesterol, TG triglycerides.

*Values are age adjusted means (95\% confidence intervals) from generalized estimating equations to account for correlated observations. Adjusted for age, propensity score, examination cycle, BMI, current smoking, hypertension, Diabetes (except for FPG) and total cholesterol (in analyses of HDL-C and TGs), using covariates from the examination in question.

${ }^{\dagger} \mathrm{mmol} / \mathrm{L}$. 
Table 6 Proportions of participants in Lipid-related categories* ${ }^{*}$ by examination cycle in large sample

\begin{tabular}{|c|c|c|c|c|c|c|c|c|c|c|}
\hline & \multicolumn{5}{|l|}{ Male } & \multicolumn{5}{|l|}{ Female } \\
\hline & $\begin{array}{l}\text { Exam } 1 \\
(1999-2001)\end{array}$ & $\begin{array}{l}\text { Exam 2 } \\
(2002-2005)\end{array}$ & $\begin{array}{l}\text { Exam } 3 \\
(2005-2008)\end{array}$ & $\begin{array}{l}\text { Exam } 4 \\
(2008-2011)\end{array}$ & $\begin{array}{l}p \text {-value } \\
\text { for trend }\end{array}$ & $\begin{array}{l}\text { Exam } 1 \\
(1999-2001)\end{array}$ & $\begin{array}{l}\text { Exam 2 } \\
(2002-2005)\end{array}$ & $\begin{array}{l}\text { Exam } 3 \\
(2005-2008)\end{array}$ & $\begin{array}{l}\text { Exam } 4 \\
(2008-2011)\end{array}$ & $\begin{array}{l}p \text {-value } \\
\text { for trend }\end{array}$ \\
\hline \multicolumn{11}{|l|}{ Age adjusted $^{+}$} \\
\hline High cholesterol & 18.3 & 14.9 & 11.7 & 9.2 & $<0.001$ & 24.8 & 22.1 & 17.3 & 14.2 & $<0.001$ \\
\hline Low HDL-C & 72.2 & 66.4 & 60.8 & 54.6 & $<0.001$ & 46.2 & 39.4 & 32.9 & 27.1 & $<0.001$ \\
\hline High Triglycerides & 33.1 & 30.1 & 27.8 & 25.4 & $<0.001$ & 26.0 & 25.2 & 22.6 & 21.0 & $<0.001$ \\
\hline High Non-HDL-C & 20.3 & 15.8 & 11.9 & 9.0 & $<0.001$ & 23.1 & 19.2 & 13.8 & 10.3 & $<0.001$ \\
\hline High TG/HDL-C & 40.2 & 44.3 & 38.4 & 25.4 & $<0.001$ & 28.3 & 25.9 & 22.3 & 19.6 & $<0.001$ \\
\hline High TC /HDL-C & 37.6 & 42.5 & 30.3 & 14.7 & $<0.001$ & 26.8 & 21.1 & 14.9 & 10.7 & $<0.001$ \\
\hline Lipid drug (\%) & 1.4 & 2.9 & 5.0 & 8.8 & $<0.001$ & 3.6 & 5.8 & 7.8 & 11.2 & $<0.001$ \\
\hline \multicolumn{11}{|l|}{ Multivariate adjusted $^{\neq}$} \\
\hline High cholesterol & 18.1 & 15.2 & 11.8 & 9.4 & $<0.001$ & 24.5 & 22.5 & 17.2 & 14.1 & $<0.001$ \\
\hline Low HDL-C & 71.0 & 67.8 & 61.2 & 53.6 & $<0.001$ & 45.4 & 40.6 & 33.1 & 26.5 & $<0.001$ \\
\hline High Triglycerides & 33.7 & 29.1 & 27.2 & 26.5 & $<0.001$ & 26.6 & 24.4 & 21.3 & 21.9 & $<0.001$ \\
\hline High Non-HDL-C & 19.9 & 16.2 & 12.1 & 9.0 & $<0.001$ & 22.9 & 19.7 & 13.6 & 10.2 & $<0.001$ \\
\hline High TG/HDL-C & 32.2 & 39.4 & 34.1 & 29.7 & $<0.001$ & 28.0 & 26.8 & 21.8 & 19.5 & $<0.001$ \\
\hline High TC /HDL-C & 41.3 & 34.4 & 26.0 & 19.1 & $<0.001$ & 26.7 & 21.8 & 14.8 & 10.6 & $<0.001$ \\
\hline Lipid drug (\%) & 1.5 & 2.9 & 4.8 & 9.0 & $<0.001$ & 3.7 & 6.1 & 7.6 & 11.4 & $<0.001$ \\
\hline
\end{tabular}

Abbreviations: Exam examination cycle, HDL-C high-density lipoprotein cholesterol, non HDL-C non-high-density lipoprotein cholesterol, TC total cholesterol, TG triglycerides.

Definitions: High cholesterol $\geq 6.19 \mathrm{mmol} / \mathrm{L} ;$ Low HDL-C $<1.036 \mathrm{mmol} / \mathrm{L}$; High Triglycerides $\geq 2.26 \mathrm{mmol} / \mathrm{L} ;$ High Non-HDL-C $\geq 5.15 \mathrm{mmol} / \mathrm{L} ; \mathrm{High} \mathrm{TG/HDL-C} \geq 2.18 ; \mathrm{High}$ TC /HDL-C $\geq 5.97$.

*Values are age and multivariate adjusted percentage from pooled logistic regressions including examination cycle to account for correlated observations.

${ }^{\dagger}$ Adjusted for age, propensity score, and examination cycle.

${ }^{\ddagger}$ Adjusted for age, propensity score, examination cycle, BMI, current smoking, hypertension, Diabetes (except for FPG) and total cholesterol (in analyses of HDL-C and TGs), using covariates from the examination in question. 
Table 7 Age-adjusted mean levels* of fasting lipids, FPG and BMI by examination cycle in small sample

\begin{tabular}{|c|c|c|c|c|c|}
\hline & Exam 1 (1999-2001) & Exam 2 (2002-2005) & Exam 3 (2005-2008) & Exam 4 (2008-2011) & $p$-value for trend \\
\hline \multicolumn{6}{|l|}{ Male } \\
\hline $\mathrm{TC}^{\dagger}$ & $5.14(5.13-5.16)$ & $5.07(5.06-5.09)$ & $5.00(4.98-5.01)$ & $4.93(4.91-4.94)$ & $<0.001$ \\
\hline $\mathrm{HDL}-\mathrm{C}^{\dagger}$ & $0.937(0.935-0.938)$ & $0.976(0.975-0.978)$ & $1.015(1.014-1.017)$ & $1.055(1.053-1.056)$ & $<0.001$ \\
\hline $\mathrm{TG}^{+}$ & $1.97(1.96-1.98)$ & $1.94(1.92-1.95)$ & $1.91(1.88-1.92)$ & $1.87(1.86-1.89)$ & $<0.001$ \\
\hline Non-HDL-C ${ }^{\dagger}$ & $4.20(4.19-4.22)$ & $4.09(4.08-4.10)$ & $3.97(3.96-3.99)$ & $3.86(3.85-3.87)$ & $<0.001$ \\
\hline TG/HDL-C & $2.33(2.31-2.34)$ & $2.21(2.19-2.22)$ & $2.08(2.07-2.10)$ & $1.96(1.95-1.98)$ & $<0.001$ \\
\hline TC/HDL-C & $5.74(5.73-5.76)$ & $5.46(5.44-5.47)$ & $5.16(5.15-5.18)$ & $4.88(4.86-4.9)$ & $<0.001$ \\
\hline $\mathrm{FPG}^{+}$ & $5.14(5.12-5.16)$ & $5.30(5.28-5.32)$ & $5.45(5.44-5.47)$ & $5.62(5.60-5.63)$ & 0.014 \\
\hline BMI $\left(\mathrm{kg} / \mathrm{m}^{2}\right)$ & $25.85(25.79-25.9)$ & $26.36(26.31-26.41)$ & $26.85(26.8-26.91)$ & $27.37(27.32-27.43)$ & $<0.001$ \\
\hline \multicolumn{6}{|l|}{ Female } \\
\hline $\mathrm{TC}^{+}$ & $5.19(5.16-5.21)$ & $5.14(5.12-5.17)$ & $5.09(5.07-5.11)$ & $5.05(5.03-5.08)$ & $<0.001$ \\
\hline HDL-C ${ }^{\dagger}$ & $1.0945(1.094-1.095)$ & $1.149(1.148-1.150)$ & $1.203(1.202-1.204)$ & $1.258(1.257-1.259)$ & $<0.001$ \\
\hline $\mathrm{TG}^{+}$ & $1.63(1.62-1.65)$ & $1.62(1.61-1.64)$ & $1.61(1.59-1.62)$ & $1.60(1.59-1.61)$ & $<0.001$ \\
\hline Non-HDL-C ${ }^{\dagger}$ & $4.09(4.07-4.11)$ & $4.00(3.97-4.02)$ & $3.89(3.88-3.91)$ & $3.80(3.77-3.82)$ & $<0.001$ \\
\hline TG/HDL-C & $1.64(1.63-1.66)$ & $1.57(1.56-1.58)$ & $1.5(1.48-1.5)$ & $1.42(1.40-1.43)$ & $<0.001$ \\
\hline TC/HDL-C & $4.97(4.95-5.00)$ & $4.72(4.70-4.74)$ & $4.46(4.44-4.48)$ & $4.21(4.19-4.23)$ & $<0.001$ \\
\hline $\mathrm{FPG}^{\dagger}$ & $5.09(5.07-5.11)$ & $5.22(5.20-5.24)$ & $5.34(5.32-5.36)$ & $5.48(5.46-5.50)$ & 0.014 \\
\hline BMI $\left(\mathrm{kg} / \mathrm{m}^{2}\right)$ & $27.43(27.35-27.5)$ & $28.16(28.08-28.23)$ & $28.81(28.74-28.9)$ & $29.50(29.43-29.58)$ & $<0.001$ \\
\hline
\end{tabular}

Abbreviations: BMI body mass index, FPG fasting plasma glucose, exam examination cycle, TC total cholesterol, HDL-C high-density lipoprotein cholesterol, non $H D L-C$ non high-density lipoprotein cholesterol, TG triglycerides.

*Values are age adjusted means ( $95 \%$ confidence intervals) from generalized estimating equations to account for correlated observations. Adjusted for age, propensity score and examination cycle. ${ }^{+} \mathrm{mmol} / \mathrm{L}$.

Cardiovascular disease has long been known to be a multi-factorial disease. In 1948, the Framingham Heart Study embarked on an ambitious project in health research to identify the common factors that contribute to cardiovascular disease [27], over the years, careful monitoring of the Framingham Study population has led to the identification of the major CVD risk factors among which lipid measures have been widely investigated [28,29]. Several initiatives have been launched in different countries to reduce the burden of CVD by reducing the level of its risk factors [30-32]. As such, the important question to be answered would be whether these findings could be translated to better risk factor levels in the population [33]. Perceiving this need, several studies have attempted to explore the time trends in the CVD risk factors in the populations of different ethnic groups. Favorable trends in the lipid measures have been documented by large studies conducted in European as well as North American populations $[28,34,35]$. However, the effects of these favorable trends on the population burden of CVD have paralleled the increasing trends in the obesity and diabetes [36,37]. As a consequence, the favorable trends in the CVD morbidity and mortality leveled off at beginning of the 21st century [11,35]. Middle Eastern population has been estimated to harbor a great fraction of the world's burden of diabetes and obesity [20,38]. In fact, while America have been estimated to spend more than half of the global health expenditure on diabetes, less than $10 \%$ of the global health expenditure will be spent in the low and middle-income countries [39]. Therefore, resorting to controlling other risk factors that are more amenable to treatment and prevention continues to be the best policy to stopping CVD. We demonstrated statistically significant and clinically meaningful favorable trends in the population levels of the lipid measures over the last decade, finding that did not change after multivariate adjustment and elimination of lipid lowering drugs users. The desirable trends for lipid measures in our population is compatible with those of other studies using cross-sectional surveys $[9-11,14]$ and prospective studies [13,16-18] as well.

We observed an increase in the population levels of HDL-C simultaneously with decline in triglycerides levels, a finding in agreement with results of the Framingham study [13] and in contrast to those of other studies $[9,10,12]$, showing simultaneous increases in TC, TGs and decreasing HDL-C. 
Table 8 Multivariate-adjusted mean levels* of fasting lipids, FPG and BMI by examination cycle in small sample

\begin{tabular}{|c|c|c|c|c|c|}
\hline & Exam 1 (1999-2001) & Exam 2 (2002-2005) & Exam 3 (2005-2008) & Exam 4 (2008-2011) & $p$-value for trend \\
\hline \multicolumn{6}{|l|}{ Male } \\
\hline $\mathrm{TC}^{\dagger}$ & $5.13(5.12-5.15)$ & $5.08(5.06-5.1)$ & $5.00(4.99-5.2)$ & $4.93(4.91-4.95)$ & $<0.001$ \\
\hline $\mathrm{HDL}-\mathrm{C}^{\dagger}$ & $0.940(0.930-0.950)$ & $0.970(0.900-0.980)$ & $1.012(1.008-1.016)$ & $1.058(1.053-1.060)$ & $<0.001$ \\
\hline $\mathrm{TG}^{+}$ & $1.97(1.95-1.99)$ & $1.95(1.93-1.98)$ & $1.91(1.88-1.94)$ & $1.89(1.86-1.91)$ & $<0.001$ \\
\hline Non-HDL-C ${ }^{\dagger}$ & $4.19(4.17-4.21)$ & $4.11(4.09-4.13)$ & $3.99(3.97-4.00)$ & $3.86(3.84-3.88)$ & $<0.001$ \\
\hline TG/HDL-C ${ }^{\dagger}$ & $2.32(2.29-2.35)$ & $2.22(2.19-2.26)$ & $2.10(2.07-2.13)$ & $1.96(1.93-1.99)$ & $<0.001$ \\
\hline $\mathrm{TC} / \mathrm{HDL}-\mathrm{C}$ & $5.72(5.69-5.75)$ & $5.48(5.45-5.51)$ & $5.19(5.16-5.22)$ & $4.86(4.83-4.90)$ & $<0.001$ \\
\hline $\mathrm{FPG}^{+}$ & $5.14(5.12-5.16)$ & $5.30(5.28-5.32)$ & $5.45(5.44-5.47)$ & $5.62(5.60-5.63)$ & 0.048 \\
\hline BMI, $\left(\mathrm{kg} / \mathrm{m}^{2}\right)$ & $25.91(25.84-25.99)$ & $26.3(26.22-26.37)$ & $26.78(26.7-26.86)$ & $27.45(27.35-27.53)$ & $<0.001$ \\
\hline \multicolumn{6}{|l|}{ Female } \\
\hline $\mathrm{TC}^{+}$ & $5.18(5.15-5.20)$ & $5.15(5.13-5.18)$ & $5.09(5.06-5.11)$ & $5.05(5.03-5.07)$ & $<0.001$ \\
\hline HDL-C ${ }^{\dagger}$ & $1.094(1.092-1.096)$ & $1.144(1.141-1.146)$ & $1.202(1.199-1.204)$ & $1.256(1.254-1.259)$ & $<0.001$ \\
\hline $\mathrm{TG}^{+}$ & $1.62(1.60-1.64)$ & $1.63(1.61-1.65)$ & $1.59(1.57-1.61)$ & $1.60(1.58-1.62)$ & $<0.001$ \\
\hline Non-HDL-C ${ }^{\dagger}$ & $4.08(4.06-4.11)$ & $4.01(3.98-4.03)$ & $3.88(3.86-3.91)$ & $3.80(3.77-3.82)$ & $<0.001$ \\
\hline $\mathrm{TG} / \mathrm{HDL}-\mathrm{C}^{\dagger}$ & $1.63(1.61-1.65)$ & $1.6(1.57-1.60)$ & $1.47(1.45-1.49)$ & $1.42(1.40-1.44)$ & $<0.001$ \\
\hline $\mathrm{TC} / \mathrm{HDL}-\mathrm{C}$ & $4.96(4.94-5.00)$ & $4.75(4.72-4.77)$ & $4.46(4.43-4.48)$ & $4.21(4.19-4.23)$ & $<0.001$ \\
\hline $\mathrm{FPG}^{\dagger}$ & $5.10(5.08-5.12)$ & $5.24(5.21-5.26)$ & $5.34(5.31-5.36)$ & $5.50(5.48-5.50)$ & 0.05 \\
\hline BMI, $\left(\mathrm{kg} / \mathrm{m}^{2}\right)$ & $27.46(27.38-27.54)$ & $28.15(28.07-28.23)$ & $28.73(28.65-28.81)$ & $29.58(29.5-29.67)$ & $<0.001$ \\
\hline
\end{tabular}

Abbreviations: BMI body mass index, exam examination cycle, FPG fasting plasma glucose, $T C$ total cholesterol, $L D L-C$ low-density lipoprotein cholesterol, $H D L-C$ high-density lipoprotein cholesterol, non HDL-C non high-density lipoprotein cholesterol, TG triglycerides.

*Values are age adjusted means (95\% confidence intervals) from generalized estimating equations to account for correlated observations. Adjusted for age, propensity score, examination cycle, BMI, current smoking, hypertension, Diabetes (except for FPG) and total cholesterol (in analyses of HDL-C and TGs), using covariates from the examination in question.

${ }^{\dagger} \mathrm{mmol} / \mathrm{L}$.

In our study the prevalence of high cholesterol decline about $48 \%$ and $42 \%$ in men and women respectively, while the percentage of US adults with high total cholesterol decline by $27 \%$ between 1999 and 2010; furthermore it was reported that about $12 \%$ of female participants and $31 \%$ of male participants had low HDL-C [14]. However, in our adult population the prevalence of low HDL-C was 52\% for men and $26 \%$ for women, despite decreasing trend in low HDL-C, dyslipidemia still has a higher prevalence, compared to U.S adults.

Although changes in nutritional habits [40,41], physical activity and endurance exercise [42,43] are all known to be among important determinants of serum lipid levels; the decreasing trends in lipid levels in our population could hardly be explained by life style changes (i.e. physical activity), since it was shown that low physical activity is common in Iranian population $[44,45]$. It has been shown, however, that over $30 \%$ of Iranian families are now consuming less hydrogenated oil than they did in the past $[46,47]$, that could possibly explain the favorable lipid trend in TLGS population during recent years. In line with our findings, cross-sectional National studies conducted by Ministry of Health and Medical Education among Iranian adult population in whole country, showed significant decrease in level of high total cholesterol (Etemad K., Center for Noncommunicable Diseases Control, Ministry of Health and Medical Education, Tehran, Iran, unpublished observations).

Another factor that affected serum lipids is cigarette smoking. Craig et al. in a meta-analysis about effect of smoking on cardiovascular risk factors demonstrated that compared with non-smokers, cigarettes smokers had significantly higher TC, TG and lower concentrations of HDL-C [48]. The review study in field of smoking between 1991 and 2007 in Iran showed that during these years smoking did not increased, which might justify the favorable trend in HDL-C level of our population [49].

Our study has both strengths and limitations. The strengths of the current study lie in its design as a long term community-based prospective study conducted on a large sample of Middle Eastern men and women, a region where data on secular trends in the lipid levels is lacking, also lipid profile components were measured rather than self-reported. Our findings need to be interpreted in light of its 
Table 9 proportions of participants in Lipid-related categories ${ }^{*}$ by examination cycle in small sample

\begin{tabular}{|c|c|c|c|c|c|c|c|c|c|c|}
\hline & \multicolumn{5}{|l|}{ Male } & \multicolumn{5}{|l|}{ Female } \\
\hline & $\begin{array}{l}\text { Exam 1 } \\
(1999-2001)\end{array}$ & $\begin{array}{l}\text { Exam } 2 \\
(2002-2005)\end{array}$ & $\begin{array}{l}\text { Exam } 3 \\
(2005-2008)\end{array}$ & $\begin{array}{l}\text { Exam } 4 \\
(2008-2011)\end{array}$ & $\begin{array}{l}p \text {-value } \\
\text { for trend }\end{array}$ & $\begin{array}{l}\text { Exam 1 } \\
(1999-2001)\end{array}$ & $\begin{array}{l}\text { Exam } 2 \\
(2002-2005)\end{array}$ & $\begin{array}{l}\text { Exam } 3 \\
(2005-2008)\end{array}$ & $\begin{array}{l}\text { Exam } 4 \\
(2008-2011)\end{array}$ & $\begin{array}{l}p \text {-value } \\
\text { for trend }\end{array}$ \\
\hline \multicolumn{11}{|l|}{ Age adjusted $^{+}$} \\
\hline High cholesterol & 15.6 & 13.2 & 11.2 & 9.4 & $<0.001$ & 18.0 & 16.4 & 14.6 & 13.4 & $<0.001$ \\
\hline Low HDL-C & 72.4 & 66.7 & 60.5 & 53.8 & $<0.001$ & 46.8 & 39.2 & 32.1 & 25.7 & $<0.001$ \\
\hline High Triglycerides & 31.2 & 27.9 & 27.3 & 24.6 & $<0.001$ & 18.3 & 17.1 & 18.1 & 16.8 & $<0.001$ \\
\hline High Non-HDL-C & 17.3 & 13.9 & 11.1 & 8.8 & $<0.001$ & 16.1 & 13.1 & 10.4 & 8.4 & $<0.001$ \\
\hline High TG/HDL-C & 42.6 & 38.0 & 33.7 & 29.4 & $<0.001$ & 21.4 & 19.5 & 17.5 & 15.9 & $<0.001$ \\
\hline High TC/HDL-C & 40.4 & 32.2 & 24.9 & 18.8 & $<0.001$ & 21.4 & 16.1 & 11.7 & 8.6 & $<0.001$ \\
\hline \multicolumn{11}{|l|}{ Multivariate adjusted $^{\neq}$} \\
\hline High cholesterol & 15.5 & 13.4 & 11.4 & 9.7 & $<0.001$ & 17.7 & 16.6 & 14.7 & 13.5 & $<0.001$ \\
\hline Low HDL-C & 71.7 & 67.0 & 60.9 & 53.0 & $<0.001$ & 42.2 & 40.0 & 32.1 & 25.4 & $<0.001$ \\
\hline High Triglycerides & 31.1 & 28.0 & 27.6 & 24.7 & $<0.001$ & 18.1 & 17.1 & 17.9 & 16.6 & $<0.001$ \\
\hline High Non-HDL-C & 17.0 & 14.2 & 11.3 & 9.0 & $<0.001$ & 15.9 & 13.4 & 10.4 & 8.5 & $<0.001$ \\
\hline High TG/HDL-C & 42.1 & 38.7 & 34.8 & 29.4 & $<0.001$ & 21.2 & 19.9 & 17.2 & 15.9 & $<0.001$ \\
\hline High TC/HDL-C & 39.8 & 33.0 & 25.4 & 18.6 & $<0.001$ & 21.2 & 16.5 & 11.7 & 8.4 & $<0.001$ \\
\hline
\end{tabular}

Abbreviations: exam examination, TC total cholesterol, HDL-C high-density lipoprotein cholesterol, non HDL-C non high-density lipoprotein cholesterol, TG triglycerides.

Definitions: High cholesterol $\geq 6.19 \mathrm{mmol} / \mathrm{L} ;$ Low HDL-C $<1.036 \mathrm{mmol} / \mathrm{L}$; High Triglycerides $\geq 2.26 \mathrm{mmol} / \mathrm{L} ;$ High Non-HDL-C $\geq 5.15 \mathrm{mmol} / \mathrm{L} ; \mathrm{High}$ TG/HDL-C $\geq 2.18 ; \mathrm{High}$ TC $/ \mathrm{HDL}-\mathrm{C} \geq 5.97$.

*Values are age and multivariate adjusted percentage from pooled logistic regressions including examination cycle to account for correlated observations.

Adjusted for age, propensity score, and examination cycle.

${ }^{\ddagger}$ Adjusted for age, propensity score, examination cycle, BMI, current smoking, hypertension, Diabetes (except for FPG) and total cholesterol (in analyses of HDL-C and TGs), using covariates from the examination in

question ${ }^{\ddagger}$ Adjusted for age, propensity score, examination cycle, BMI, current smoking, hypertension, Diabetes (except for FPG) and total cholesterol (in analyses of HDL-C and TGs), using covariates from the examin-

ation in question. 
limitations as is inherent to any prospective study [13]. First, survivor bias might have biased favorable trends towards overestimated values, i.e. individuals with possible unfavorable changes in their lipid levels might have died and thus been excluded from repeated measurements. Second, as any cohort study we cannot rule out healthy cohort effect i.e. the possibility of the effect of knowledge about the serum lipids might have affected the lifestyle or lipid drugs consumption in the participants, leading to the favorable trends in lipid levels. Third, we did not have any systematic data on the trends of nutritional behavior, physical activity and knowledge of the primary prevention in our population, consequently it is not possible to test the hypothesis that whether the trends observed could be attributable to changes in physical activity or nutrition status. Forth, the results obtained in the current study might not applicable to certain age groups including younger (less than 20 years) and older (over than 75 years) ones. Finally, our population was selected from middleaged Middle East Caucasians and therefore we cannot make inferences beyond a similar group.

\section{Conclusion}

The main findings in this study include an observed decrease in total cholesterol, LDL-C, triglyceride levels as well as TC/HDL-C and TG/HDL-C in an adult Iranian population during the period of 19992001 until 2008-2011.Overall the trends of general obesity and FPG level were observed to have increased. The net effect of such trends on the CVD burden warrants further investigations.

\section{Competing interests}

The authors declare that they have no competing interests

\section{Authors' contributions}

Conceived and designed the experiments: FH, MK. Analyzed the data: SA. Wrote the paper: MK, FH, ML, MB, involved in acquisition of analysis data files from cohort database: SA, Contributed to interpretation of results: FH, MK, NS, MT, FA, ML, MB. Critically reviewed drafts of the manuscript and made comments to improve clarity: MK, FH, ML, and MT. All authors read and approved the final manuscript.

\section{Funding}

This study was supported by grant No. 121 from the National Research Council of Iran. We express appreciation to Mrs. Niloofar Shiva for their editorial contribution and constructive feedback. The funding source had no role in design, in the collection, analysis, and interpretation of data; in the writing of the manuscript; and in the decision to submit the manuscript for publication.

\section{Author details}

${ }^{1}$ Prevention of Metabolic Disorders Research Center, Research Institute for Endocrine Science, Shahid Beheshti University of Medical Sciences, \#24, Parvaneh st, Yemen st, Chamran Exp, P.O. Box: 19395-4763, Tehran, Islamic Republic of Iran. 'Endocrine Research Center, Research Institute for Endocrine sciences, Shahid Beheshti University of Medical Sciences, Tehran, Iran.
Received: 17 December 2013 Accepted: 16 January 2014

Published: 23 January 2014

\section{Reference}

1. Brown JR, O'Connor GT: Coronary heart disease and prevention in the United States. N Engl J Med 2010, 362:2150-2153.

2. Hatmi Z, Tahvildari S, Motlag AG, Kashani AS: Prevalence of coronary artery disease risk factors in Iran: a population based survey. BMC CardiovasC Disord 2007, 7:32.

3. Ford ES, Capewell S: Coronary heart disease mortality among young adults in the U.S. from 1980 through 2002: concealed leveling of mortality rates. J Am Coll Cardiol 2007, 50:2128-2132.

4. Wilson PW, Garrison RJ, Castelli WP, Feinleib M, McNamara PM, Kannel WB: Prevalence of coronary heart disease in the Framingham Offspring Study: role of lipoprotein cholesterols. Am J Cardiology 1980, 46:649-654.

5. Assmann G, Schulte $H$, von Eckardstein A, Huang Y: High-density lipoprotein cholesterol as a predictor of coronary heart disease risk. The PROCAM experience and pathophysiological implications for reverse cholesterol transport. Atherosclerosis 1996, 124(Suppl):S11-S20.

6. Austin MA, Hokanson JE, Edwards KL: Hypertriglyceridemia as a cardiovascular risk factor. Am J Cardiol 1998, 81:7B-12B.

7. Azizi F, Rahmani M, Ghanbarian A, Emami H, Salehi P, Mirmiran P, Sarbazi N: Serum lipid levels in an Iranian adults population: Tehran Lipid and Glucose Study. Eur J Epidemiol 2003, 18:311-319.

8. Tohidi M, Mohebi R, Cheraghi L, Hajsheikholeslami F, Aref S, Nouri S, Azizi F, Hadaegh F: Lipid profile components and incident cerebrovascular events versus coronary heart disease; the result of 9 years follow-up in Tehran Lipid and Glucose Study. Clin Biochem 2013, 46:716-721.

9. Gupta R, Guptha S, Agrawal A, Kaul V, Gaur K, Gupta VP: Secular trends in cholesterol lipoproteins and triglycerides and prevalence of dyslipidemias in an urban Indian population. Lipids Health Dis 2008, 7:40

10. Arai H, Yamamoto A, Matsuzawa Y, Saito Y, Yamada N, Oikawa S, Mabuchi $\mathrm{H}$, Teramoto T, Sasaki J, Nakaya N, et al: Serum lipid survey and its recent trend in the general Japanese population in 2000. J Atheroscler Thromb 2005, 12:98-106.

11. Arnett DK, Jacobs DR Jr, Luepker RV, Blackburn H, Armstrong C, Claas SA: Twenty-year trends in serum cholesterol, hypercholesterolemia, and cholesterol medication use: the Minnesota Heart Survey, 1980-1982 to 2000-2002. Circulation 2005, 112:3884-3891.

12. Li JZ, Wang S, Dong J, Zhu LH, Li YL, Gao DL, Tian YP, Dong L, Yang L, Li SK: Present status of serum lipid levels in Beijing professional populations and its trend of changes over 15 years-a collaborative study of seven research and clinical laboratories in Beijing. Clinica chimica acta; international journal of clinical chemistry 2005, 352:199-207.

13. Ingelsson E, Massaro JM, Sutherland P, Jacques PF, Levy D, D'Agostino RB, Vasan RS, Robins SJ: Contemporary trends in dyslipidemia in the Framingham Heart Study. Arch Ind Health 2009, 169:279-286.

14. Carroll MD, Kit BK, Lacher DA: Total and high-density lipoprotein cholesterol in adults: National Health and Nutrition Examination Survey, 2009-2010. NCHS data brief 2012, 1:1-8.

15. Bozorgmanesh MR, Hadaegh F, Padyab M, Mehrabi Y, Azizi F: Temporal changes in anthropometric parameters and lipid profile according to body mass index among an adult Iranian urban population. Ann Nutr Metab 2008, 53:13-22.

16. Szklo M, Chambless LE, Folsom AR, Gotto A Jr, Nieto FJ, Patsch W, Shimakawa T, Sorlie P, Wijnberg L: Trends in plasma cholesterol levels in the atherosclerosis risk in communities (ARIC) study. Prev Med 2000, 30:252-259.

17. Abbott RD, Yano K, Hakim AA, Burchfiel CM, Sharp DS, Rodriguez BL, Curb JD: Changes in total and high-density lipoprotein cholesterol over 10- and 20-year periods (the Honolulu Heart Program). Am J Cardiol 1998, 82:172-178.

18. Ferrara A, Barrett-Connor E, Shan J: Total, LDL, and HDL cholesterol decrease with age in older men and women. The Rancho Bernardo Study 1984-1994. Circulation 1997, 96:37-43.

19. Farzadfar F, Finucane MM, Danaei G, Pelizzari PM, Cowan MJ, Paciorek CJ, Singh GM, Lin JK, Stevens GA, Riley LM: National, regional, and global trends in serum total cholesterol since 1980: systematic analysis of health examination surveys and epidemiological studies with 321 country-years and 3. 0 million participants. Lancet 2011, 377:578-586. 
20. Harati H, Hadaegh F, Saadat N, Azizi F: Population-based incidence of Type 2 diabetes and its associated risk factors: results from a six-year cohort study in Iran. BMC public health 2009, 9:186

21. Azizi F, Ghanbarian A, Momenan AA, Hadaegh F, Mirmiran P, Hedayati M Mehrabi Y, Zahedi-Asl S, Tehran L, Glucose Study G: Prevention of noncommunicable disease in a population in nutrition transition: Tehran Lipid and Glucose Study phase II. Trials 2009, 10:5.

22. Friedewald WT, Levy RI, Fredrickson DS: Estimation of the concentration of low-density lipoprotein cholesterol in plasma, without use of the preparative ultracentrifuge. Clinical chemistry 1972, 18:499-502.

23. Expert Panel on Detection E, Treatment of High Blood Cholesterol in A: Executive Summary of The Third Report of The National Cholesterol Education Program (NCEP) Expert Panel on Detection, Evaluation, And Treatment of High Blood Cholesterol In Adults (Adult Treatment Panel III). JAMA 2001, 285:2486-2497.

24. Jr DAR: Propensity score methods for bias reduction in the comparison of a treatment to a non-randomized control group. Stat Med 1998, 17:2265-2281.

25. Rees K, Dyakova M, Ward K, Thorogood M, Brunner E. (Eds.): Dietary advice for reducing cardiovascular risk. 2013.

26. Molenberghs G, Verbeke G: Models for discrete longitudinal data. New York. USA: Springer; 2005.

27. Cupples LA, Yang Q, Demissie S, Copenhafer D, Levy D, Framingham Heart Study I: Description of the Framingham Heart Study data for Genetic Analysis Workshop 13. BMC Genet 2003, 4(Suppl 1):S2.

28. Sytkowski PA, Kannel WB, D'Agostino RB: Changes in risk factors and the decline in mortality from cardiovascular disease. The Framingham Heart Study. N Engl J Med 1990, 322:1635-1641.

29. Hubert HB, Feinleib M, McNamara PM, Castelli WP: Obesity as an independent risk factor for cardiovascular disease: a 26-year follow-up of participants in the Framingham Heart Study. Circulation 1983, 67:968-977.

30. De Backer G, Ambrosioni E, Borch-Johnsen K, Brotons C, Cifkova R, Dallongeville J, Ebrahim S, Faergeman O, Graham I, Mancia G, et al: European guidelines on cardiovascular disease prevention in clinical practice. Third Joint Task Force of European and Other Societies on Cardiovascular Disease Prevention in Clinical Practice. Eur Heart J 2003, 24:1601-1610.

31. Graham I, Atar D, Borch-Johnsen K, Boysen G, Burell G, Cifkova R, Dallongeville J, De Backer G, Ebrahim S, Gjelsvik B, et al: European guidelines on cardiovascular disease prevention in clinical practice: full text. Fourth Joint Task Force of the European Society of Cardiology and other societies on cardiovascular disease prevention in clinical practice (constituted by representatives of nine societies and by invited experts). European journal of cardiovascular prevention and rehabilitation : official journal of the European Society of Cardiology, Working Groups on Epidemiology \& Prevention and Cardiac Rehabilitation and Exercise Physiology 2007, 14(Suppl 2):S1-S113.

32. Perk J, De Backer G, Gohlke H, Graham I, Reiner Z, Verschuren M, Albus C, Benlian P, Boysen G, Cifkova R, et al: European Guidelines on cardiovascular disease prevention in clinical practice (version 2012). The Fifth Joint Task Force of the European Society of Cardiology and Other Societies on Cardiovascular Disease Prevention in Clinical Practice (constituted by representatives of nine societies and by invited experts). Eur Heart J 2012, 33:1635-1701.

33. Uemura K, Pisa Z: Trends in cardiovascular disease mortality in industrialized countries since 1950. World health statistics quarterly Rapport trimestriel de statistiques sanitaires mondiales 1987, 41:155-178.

34. Vartiainen E, Laatikainen T, Peltonen M, Juolevi A, Mannisto S, Sundvall J, Jousilahti P, Salomaa V, Valsta L, Puska P: Thirty-five-year trends in cardiovascular risk factors in Finland. Int J Epidemiol 2010, 39:504-518.

35. Arnett DK, McGovern PG, Jacobs DR Jr, Shahar E, Duval S, Blackburn H, Luepker RV: Fifteen-year trends in cardiovascular risk factors (1980-1982 through 1995-1997): the Minnesota Heart Survey. Am J Epidemiol 2002, 156:929-935.

36. Gregg EW, Cheng YJ, Cadwell BL, Imperatore G, Williams DE, Flegal KM, Narayan $\mathrm{KM}$, Williamson DF: Secular trends in cardiovascular disease risk factors according to body mass index in US adults. JAMA 2005, 293:1868-1874.

37. Lopez AD, Mathers CD, Ezzati M, Jamison DT, Murray CJ: Global and regional burden of disease and risk factors, 2001: systematic analysis of population health data. Lancet 2006, 367:1747-1757.

38. Motlagh B, O'Donnell M, Yusuf S: Prevalence of cardiovascular risk factors in the Middle East: a systematic review. European journal of cardiovascular prevention and rehabilitation: official journal of the European Society of Cardiology, Working Groups on Epidemiology \& Prevention and Cardiac Rehabilitation and Exercise Physiology 2009, 16:268-280.
39. Zhang P, Zhang X, Brown J, Vistisen D, Sicree R, Shaw J, Nichols G: Global healthcare expenditure on diabetes for 2010 and 2030. Diabetes Res Clin Pract 2010, 87:293-301

40. Nakamura H, Arakawa K, Itakura H, Kitabatake A, Goto Y, Toyota T, Nakaya N, Nishimoto S, Muranaka M, Yamamoto A, et al: Primary prevention of cardiovascular disease with pravastatin in Japan (MEGA Study): a prospective randomised controlled trial. Lancet 2006, 368:1155-1163.

41. Brunner EJ, Rees K, Ward K, Burke M, Thorogood M: Dietary advice for reducing cardiovascular risk. Cochrane Database Syst Rev 2007, Art. No. CD002128(Issue 4). DOI: 10.1002/14651858.CD002128.pub3.

42. Varady KA, Jones PJ: Combination diet and exercise interventions for the treatment of dyslipidemia: an effective preliminary strategy to lower cholesterol levels? J Nutr 2005, 135:1829-1835.

43. Wilund KR, Feeney LA, Tomayko EJ, Weiss EP, Hagberg JM: Effects of endurance exercise training on markers of cholesterol absorption and synthesis. Physiol Res 2009, 58:545-552.

44. Esteghamati A, Khalilzadeh O, Rashidi A, Kamgar M, Meysamie A, Abbasi M: Physical activity in Iran: results of the third national surveillance of risk factors of non-communicable diseases (SuRFNCD-2007). J Phys Act Health 2011, 8:27-35

45. Momenan AA, Delshad M, Mirmiran P, Ghanbarian A, Azizi F: Leisure Time Physical Activity and Its Determinants among Adults in Tehran: Tehran Lipid and Glucose Study. Int J Prev Med 2011, 2:243-251.

46. Torabi P, Zare F, Shekholeslam R, Safavi SM: Study of vegetable oil consumption in 15 pilot universities of Iran, In 12th Nutritional Congress. Tabriz; Iran; 2004

47. Mohammadifard N, Toghianifar N, Sajjadi F, Alikhasi H, Kelishadi R, Maghroun M, Esmaeili M, Ehteshami S, Tabaie H, Sarrafzadegan N: Improvement of dietary oil consumption following a community trial in a developing country: the role of translational research in health promotion. ARYA atherosclerosis 2013, 9:29-37.

48. Craig WY, Palomaki GE, Haddow JE: Cigarette smoking and serum lipid and lipoprotein concentrations: an analysis of published data. BMJ (Clinical research ed) 1989, 298:784-788.

49. Meysamie A, Ghaletaki R, Zhand N, Abbasi M: Cigarette Smoking in Iran Iranian J Publ Health 2012, 41:1-14.

\section{doi:10.1186/1476-511X-13-20}

Cite this article as: Kheirandish et al: Secular trends in serum lipid levels of a Middle Eastern adult population; 10 years follow up in Tehran lipid and glucose study. Lipids in Health and Disease 2014 13:20.

\section{Submit your next manuscript to BioMed Central and take full advantage of:}

- Convenient online submission

- Thorough peer review

- No space constraints or color figure charges

- Immediate publication on acceptance

- Inclusion in PubMed, CAS, Scopus and Google Scholar

- Research which is freely available for redistribution

Submit your manuscript at www.biomedcentral.com/submit
C) BioMed Central 